\title{
EZH2 Promotes Extracellular Matrix Degradation via Nuclear Factor-kB(NF-kB)and p38 signaling Pathways in Pulpitis.
}

jie He

Peking university school and hospital of stomatology

Man Qin

Peking University - School of Stomatology: Peking University School of Stomatology

\section{Yingyi Chen}

Peking University - School of Stomatology: Peking University School of Stomatology

\section{Ziqi Hu}

Peking University - School of Stomatology: Peking University School of Stomatology

\section{Ling Ye}

Sichuan University Huaxi College of Stomatology: Sichuan University West China College of

Stomatology

Tianqian Hui ( $\square$ huitianqian@sina.com )

Peking University School of Stomatology https://orcid.org/0000-0002-4359-211X

\section{Research}

Keywords: EZH2,pulpitis, extracellular matrix, Nuclear Factor-kB, P38

Posted Date: December 29th, 2020

DOI: https://doi.org/10.21203/rs.3.rs-134982/v1

License: (c) (1) This work is licensed under a Creative Commons Attribution 4.0 International License. Read Full License 


\section{Abstract}

Background: Pulpitis is a complicated chronic inflammatory process which in a dynamic balance between damage and repair. Extracellular matrix plays an important regulatory role in wound healing and tissue repair. The aim of this study was to explore role of the epigenetic mark, enhancer of zeste homolog 2 (EZH2) on the degradation of extracellular matrix during pulpitis.

Methods : Quantitative polymerase chain reaction was used to assess the expression of matrix metalloproteinases (MMPs) and type I collagen in HDPCs upon EZH2 and El1 stimulation. The mechanism of EZH2 affecting extracellular matrix was explored through quantitative polymerase chain reaction and Western blot. A rat model of dental pulp inflammation was established, and the expression of type I collagen in dental pulp under EZH2 stimulation was detected by immunohistochemical staining.

Results :EZH2 upregulated the expression of MMP-1, MMP-3, MMP-8 and MMP-10 and decreased the production of type I collagen in HDPCs, while El1 had the opposite effect .EZH2 activated the Nuclear Factor-kB(NF-kB)and p38 signaling Pathways in HDPCs, the inhibition of which reversed the induction of MMPs and the suppression of type I collagen .EZH2 can downregulated the type I collagen levels in an experimental model of dental pulpitis in rats.

Conclusion: EZH2 promotes extracellular matrix degradation via Nuclear Factor-kB(NF-kB)and P38 signaling pathways in pulpitis.EZH2 can decrease the type I collagen levels in vivo and vitro.

\section{Introduction}

Dental pulp is derived from ectodermal stroma and is a loose connective tissue rich in extracellular matrix (ECM). Type I and type III collagen is the primary constituent of the ECM of dental pulp[1]. Pulpitis is one of the common dental disorders associated with tooth pulp inflammation[2]. In pulpitis tissue, HDPCs, the main dental pulp component, can interact with immune cells to secrete significant amounts of inflammatory cytokines and chemokines locally. These cytokines and chemokines may promote pulpitis progression, tissue destruction, or regulate inflammatory responses to eliminate pathogens[3, 4]. Recent studies have proved that ECM plays an important regulatory role in cell proliferation, differentiation, wound healing and tissue repair[5]. ECM is closely related to the repair function of dental pulp tissue, and participates in and regulates this process, such as participating in and mediating the migration and differentiation of dental pulp cells, becoming a solid support for newly generated dentin cells and taking part in the initiation of dentin mineralization [6]. As extracellular matrix plays an important role in the progress and repair of pulpitis, studying the dynamic changes of extracellular matrix in pulpitis will help reveal the mechanism of pulpitis and provide theoretical basis for the treatment of pulpitis.

Previous studies have demonstrated that the endopeptidase activity of matrix metalloproteinase (MMP) mediates the degradation of ECM, which may promote the progress of pulpitis[7]. The activity of MMP against extracellular matrix substrates is regulated at 4 "gates": 1) by transcriptional regulation of MMP genes; 2 ) by precursor activation; 3 ) by differences in substrate specificity; and 4) by MMP inhibitors[8]. 
Existing research attempts to reveal the characteristics of transcription factors that control the active promoter of the MMP gene, but the transcription mechanism that regulates MMP during the progression of inflammatory diseases is unclear, such as epigenetic regulation[9]. In inflammatory diseases such as rheumatoid arthritis, tuberculosis, and diabetes, epigenetics (including DNA methylation, histone modifications, and non-coding RNA) can regulate the expression of MMP and affect the progress of inflammatory diseases[10] .

At present, it has been reported that the histone methylation of H3K27 may be involved in dental pulp inflammation and repair process [11]. Studies have shown that the reduced expression of H3K27me3 in inflammatory dental pulp tissues and dental pulp cells may induce the regeneration process of dental pulp[11]. In addition, Jumonji domain protein 3 (JMJD3) is an enzyme that eliminates the histone H3K27

methylation marker and can regulate the expression and transcriptional activity of H3K27me3. Recently, it was discovered that Jmjd3 can regulate the differentiation and cell characteristics of macrophages, suggesting that it may provide a link between inflammation and epigenome reprogramming[12].

In addition, the methyltransferase of H3K27, Enhancer of zeste homolog $2(\mathrm{EZH} 2)$ can be used as a regulator of pulp inflammation[11]. Our previous research found that during the development of dental pulp inflammation, the expression of EZH2 increased[11]. Existing studies suggest that EZH2 is involved in the repair process of dental pulp damage, but whether this process involves extracellular matrix is unclear, and whether EZH2 can affect dental pulp inflammation by regulating MMPs remains to be studied.

It is known that in some circumstances nuclear factor kappa B (NF-kB) and MAPK signaling pathways are required for the induction of histone modifiers [13]. These NF-kB and MAPK signaling pathways are also known to be major vehicles for producing inflammation[14]. In this study, we addressed the following hypothesis: EZH2 affects the expression of MMP in dental pulp through the NF-kB pathway or MAPK pathway, thereby mediating the degradation of extracellular matrix and affecting the progress of dental pulp inflammation such as the collagen degradation.

\section{Results}

\section{Immunohistochemistry to identify dental pulp cells}

The HDPCs were fusiform in shape (Fig. 1A-1B) and showed positive immunostaining for vimentin(Fig. 1B), but negative immunostaining for cytokeratin14(Fig. 1A).

\section{EZH2 influenced ECM balance of HDPCs}

After EZH2 stimulated HDPC for $2 h, 4 h$, and $24 h$, we quantified the mRNA levels of the MMPs and type I collagen. The expression of MMP-1DMMP-3ロMMP-8 and MMP-10 were increased than the control group 
$(P<0.05)$ (Figure 2A-2D). While the expression of MMP-2 and MMP-13 both had a decrease $(P<0.001)$

(Figure 2E-F); There was a significant decrease in the expression of type I collagen $(P<0.01)$ (Figure $2 \mathrm{H})$.

\section{EZH2 promotes ECM degradation via NF-kB and p38 signaling Pathways in HDPCs}

To gain further insight into the mechanism underlying EZH2-induced cytokine/ chemokine expression in HDPCs, we examined the NF-kB and MAPK pathways, which have been suggested by previous reports to be involved in modulating cytokine/chemokine expression $[15,16]$.EZH2 treatment led to the activation of the MAPK and NF-kB pathways, as demonstrated by phosphorylation of those signaling molecules after 15-45 min of stimulation(Fig.3A). When we treated the HDPC with NF-kB inhibitor (BAY11-7082) or specific p38(SB203580) inhibitor, the expression of phosphorylation of those signaling molecules were decreased(Fig.3B-C). The EZH2 -mediated induction of MMP-10MMP-3ロMMP-8 and MMP-10 expression decreased significantly by treatment with an NF-kB inhibitor (BAY11-7082) or p38(SB203580) inhibitors in 2 hours (Fig. 3D-G). On the contrary, EZH2 -mediated reduction of type I collagen expression increased significantly by treatment with an NF-kB inhibitor (BAY11-7082) or p38(SB203580) inhibitors in 2 hours (Fig. 3H).

\section{EZH2 and El1 effect the development of pulpitis}

After El1 stimulated HDPC for $2 \mathrm{~h}, 4 \mathrm{~h}$, and $24 \mathrm{~h}$, the change of MMP-3 had no statistical difference in the first two groups, but declined after $24 \mathrm{~h}(\mathrm{P}<0.0001)$ (Figure $3 \mathrm{~A})$; the expression of MMP-8 was declined $(P<0.001)$ (Figure 3B); the expression of MMP-2 began to decrease after $24 \mathrm{~h}(\mathrm{p}<0.5)$ (Figure $3 \mathrm{C})$. The expressions of MMP-1, MMP-10, and MMP-13 all increased after El1 stimulation ( $\mathrm{P}<0.001)$ (Figure 3D-F); the mRNA levels of type I collagen had a remarkable increase at $2 \mathrm{~h}$ and $24 \mathrm{~h}$ (Figure $3 \mathrm{H}$ ).

To examine Col-1 change during pulpitis in vivo, we established a pulpitis model based on experiments with rats. Pulp tissue samples were stained with hematoxylin-eosin stain to verify the infection. We detected inflammatory cell infiltration . Immunohistochemistry staining of Col-1 further revealed that $E Z H 2$ can reduce the expression of Col- 1 in inflammatory dental pulp tissue( $4 \mathrm{~J}, \mathrm{~K}, \mathrm{M}$ and $\mathrm{N}$ ). However, El1did not increase the expression of Col-1 significantly (4L and 0 ).

\section{Discussion}

In the experiment, HDPCs were further subjected to immunocytochemical detection of cell sources. Postnatal expression of vimentin is restricted to fibroblasts, endothelial cells, lymphocytes, and several specialized cells of the thymus and the brain[17]. Cytokeratins (CK) are abundant in keratinized cells, particularly CK14 and CK19, which are expressed in stratified squamous epithelial cells[18]. In our experiment, we showed that the cells were positive for vimentin and negative for cytokeratin 14 , which 
proved that the cells were mesoderm derived and there were no epithelial cells mixed, which was consistent with the biological characteristics of HDPCs.

Our experiment verified that EZH2 could increase the expression of MMP-1, MMP-3,MMP-8 and MMP-10 and (Fig. 2). MMP-1 (Collagenase 1, fibroblast collagenase) is an essential enzyme responsible for degrading type I and type III collagen since the initial breakdown of these fibrillar collagen network is mediated primarily by MMP-1 $[19,20]$. In Su-Jung Shin's experiment, the concentrations of MMP-1 in acute-inflamed pulps and chronic-inflamed pulps groups were significantly higher than in the control[21]. According to Bergenholtz G, MMP-8 in pulpal inflammatory lesions is mainly of PMN origin[22].PMNs are cells forming pulp abscesses, and the main MMP-8 positivity was accumulated around the pulp abscess suggesting that it also contributes to the tissue destruction of pulp necrosis and abscesses. Previous studies have revealed that PMN-leucocytes, macrophages and plasma cell produced MMP-8 in pulp and periapical granulomas[23]. Evrosimovska B, et al. confirmed that degradation of the collagen of the organic matrix from pulp tissue during chronic inflammation is an enzyme process and that collagenses (MMP-1, -8) are invovlved in these destructive process[24]. Rhim EM et al. found the expression of MMP$3,-10$ was up-regulated in the pulp cells after 24 hours of stimulation with TNF-a[25].In our study, EZH2 suppressd the production of type I collagen in HDPCs. Type I and III collagens are the most abundant collagens in dental pulp, and collagen type I expression is thought to have a direct effect on osteogenic differentiation and mineralization[26]. During the process of inflammation, reduced collagen synthesis and increased degradation led to disintegration of ECM and remodeling of dental pulp[27].Our experiment suggested that EZH2 may affect the development of Pulpitis by regulating the expression of MMPs, but the specific mechanism is not clear, so we did further experiment.

In our study, the EZH2-induced inflammation response in HDPCs appears to represent a collaboration of the NF-kB, and MAPK pathways. EZH2 stimulated the phosphorylation of MAPK and NF-kB, whereas inhibition of MAPK or NF-kB by specific inhibitors induced a dramatic reduction in EZH2-induced MMPs production, suggesting that they all have some roles in EZH2-mediated inflammation (Fig. 3). These results are in accordance with previous studies showing that ASH1L (absent, small, or homeotic 1-like, an H3K4 methyltransferase that can antagonize EZH2) significantly upregulate the expression of MMP-1, MMP-2 and MMP-13 through the Mitogen-activated Protein Kinase (MAPK) signaling pathway[16].In the rat experimental pulp infection model, EZH2 decreased the expression of Col-1, but El1 could not upregulate the expression of Col-1 in rat pulpitis. El1 could increase the expression of Col-1 in vitro, suggesting that the development of pulpitis is a multifactor regulatory process, and inhibition of EZH2 alone is not enough to promote the repair of collagen fibers.

During pulpitis, pulp can be in a dynamic balance between damage and repair response[28, 29]. In recent years, studies have shown that ECM plays an important regulatory role in wound healing and tissue repair[5]. Exploring the dynamic changes of extracellular matrix in dental pulp inflammation will provide a theoretical basis for the treatment of pulpitis. Early studies have found that epigenetic regulatory factor $\mathrm{EZH} 2$ is involved in the process of dental pulp inflammation and repair[11],but whether this process involves extracellular matrix is not clear. This study verified that EZH2 affects the expression of MMP in 
dental pulp through the NF-kB pathway or MAPK pathway(Fig. 3), thereby mediating the degradation of extracellular matrix and affecting the progress of dental pulp inflammation.

Inflammation can stimulate the production of MMPs with subsequent ECM remodeling[8]. Odontoblasts secrete some of the essential MMPs for both physiologic and pathologic conditions. MMPs also appear to be a participant in the process of reversible and irreversible pulpitis[6].

\section{Conclusions}

EZH2 promotes extracellular matrix degradation via Nuclear Factor-kB(NF-kB)and P38 signaling pathways in pulpitis.EZH2 can decrease the type I collagen levels in vivo and vitro. The down-regulation of EZH2 promotes the development of inflammatory pulp to repair reaction, and provides new ideas for the treatment of pulpitis.

\section{Material And Methods}

\section{Cell culture and treatment}

Primary HDPCs were collected from the caries-free third molars and the premolars that need to be extracted for orthodontics of healthy donors in maxillofacial surgery clinic of Peking University School of Stomatology (ethics approval number: PKUSSIRB-201732003). Cells were maintained in a-MEM medium (Gibco, USA) with 10\% fetal calf serum (FBS) (Excell Bio, Australia) and 100U/ml penicillin and 100 $\mu \mathrm{g} / \mathrm{ml}$ streptomycin sulfate, and passages 3-5 were used. Cells were treated with recombinant $\mathrm{EZH} 2(2 \mathrm{ng} / \mathrm{mL}$,abnova,USA) or the inhibitor of EZH2 El1 (2uMol/L APExBIO,abnova,USA) for the given times. In the specified experiments, cells were pretreated with one of the following specific pathway inhibitors: BAY11-7082(an Ik B phosphorylation inhibitor, 1 uM , Selleckchem , Houston, TX) or SB203580(a p38 MAPK inhibitor,20 uM, Cell Signaling Technology, Danvers, MA).

\section{Characterization of HDPCs}

The fourth-generation HDPC was inoculated in a 12-well plate and grown in slides. After the cells grew close to confluence, they were fixed with $4 \%$ paraformaldehyde. Primary antibodies included vimentin(1:1000; proteintech , USA) and cytokeratin 14 (1:1000; proteintech ,USA ). A standard immunohistochemistry kit (Zhongshanjinqiao, Beijing, China) was used for immunohistochemistry. After counterstaining with hematoxylin and dehydration, the expression of related antibodies was observed.

\section{RNA Isolation and Reverse Transcription and Real-time PCR}

Total RNA of the HDPCs was extracted by TRIzol reagent (Invitrogen, Carlsbad, CA). RNA concentrations were measured using a Nanodrop Spectrophotometer (Thermo Fisher Scientific). Complementary DNA 
was synthesized from 1000 ng RNA using a PrimeScript RT kit (TaKaRa, Dalian, China). The produced complementary DNA was prepared as templates for the polymerase chain reaction using SYBR Premix Ex Taq (Takara) according to the manufacturer's instruction. Conditions were applied as following: $50^{\circ} \mathrm{C}$ for 2 min, then $95^{\circ} \mathrm{C}$ for $10 \mathrm{~min}$, followed by 40 cycles of $94^{\circ} \mathrm{C}$ for $15 \mathrm{~s}$ and $60^{\circ} \mathrm{C}$ for 1 min using an $\mathrm{ABI}$ PRISM 7500 Sequence Detection System (Applied Biosystems, Foster City, CA, USA). The Ct values obtained from different samples were compared using the $2^{-\Delta \Delta C t}$ method. Glyceraldehyde-3-phosphate dehydrogenase (GAPDH) served as an internal reference gene. .Primers were designed to generate products of less than $200 \mathrm{bp}$ for efficient analysis and were as follows:MMP-1,5'-

AGATTCTACATGCGCACAAATC-3' (forward) and 5'-CCTTTGAAAAACCGGACTTCAT-3'(reverse);MMP-2,5'CAACTACAACTTCTTCCCTCGCA-3' (forward) and 5'-GGTCACATCGCTCCAGACTTG-3' (reverse); MMP-3,5'GAGGACACCAGCATGAACCT-3' (forward) and 5'-CACCTCCAGAGTGTCGGAGT-3' (reverse);MMP-8,5'CCAACTATGCTTTCAGGGAAAC-3' (forward) and 5'- GTTGGATAGGGTTGCTTGAAAG-3' (reverse);MMP10,5'-TTGCCCAGCAATACCTAGAAAA-3' (forward) and 5'-GAACTTCTGCATTCCTTGGATT- 3' (reverse);MMP-13,5'-GCACTTCCCACAGTGCCAT -3'(forward) and 5'-AGTTCTTCCCTTGATGGCCG-3' (reverse) ;COL1A1,5'-GCTCGTGGAAATGATGGTGC-3' (forward) and 5'-ACCCTGGGGACCTTCAGAG-3' (reverse); GAPDH,5'-TCAACAGCGACCCACTC-3' (forward) and 5'-GCTCTAGCCAAATTCGTTGTC-3' (reverse).

\section{Western Blot Analysis}

The cells were lysed using RIPA buffer(Solarbio,China) supplemented with protease inhibitors (Solarbio,China). Cell homogenates were obtained by gently scraping the cells from each well, and the protein concentrations from the lysates were determined by using the BCA Protein Assay Kit (ThermoScientific, Rockford,IL). Prior to loading, total protein samples were denatured by heating at $95^{\circ} \mathrm{C}$ for $10 \mathrm{~min}$ in $5 \mathrm{x}$ SDS-PAGE sample loading buffer (Applygen, China). 30 $\mathrm{\mu g}$ of the protein sample were separated by SDS-polyacrylamide gel electrophoresis at $110 \mathrm{~V}$ for $90 \mathrm{~min}$ and transferred to $0.45-\mu \mathrm{m}$ polyvinylidene fluoride (PVDF) membranes at a constant current of $300 \mathrm{~mA}$ for $60 \mathrm{~min}$. After blocking in $5 \%$ nonfat dry milk (Bioruler, China) at room temperature, the membranes were incubated with rabbit antiphospho-p65 (catalogno.3033, Cell Signaling Technolog, Danvers,MA), rabbit anti -p65 (catalogno.8242, Cell Signaling Technolog, Danvers,MA), rabbit anti -phospho-p38 (catalogno. 4511, Cell Signaling Technolog, Danvers,MA), rabbit anti- p38(catalogno.9212, Cell Signaling Technolog, Danvers,MA) overnight at $4^{\circ} \mathrm{C}$. The membrane was incubated for $1 \mathrm{~h}$ at room temperature with horseradish peroxidase (HRP)-conjugated anti-rabbit immunoglobulin(Proteintech,USA) based on the source of the corresponding primary antibody, and the immunoblots were detected using a Western enhanced chemiluminescence blotting kit (ECL, SOLIBRO, China).

\section{Construction of Rat Experimental Pulp Infection Model}


12 male 6-week-old SD rats (Weitong Lihua Company, China) were subdivided into 4 groups of 3 rats each.Rats were classified according to the sealing medicine: LPS $(10 \mathrm{mg} / \mathrm{ml}$, Sigma, USA) for group1, LPS $(10 \mathrm{mg} / \mathrm{ml})+\mathrm{EZH} 2(20 \mathrm{ng} / \mathrm{mL})$ for group2, LPS $(10 \mathrm{mg} / \mathrm{ml})+\mathrm{El} 1(20 \mathrm{ng} / \mathrm{mL})$ for group3 and group4 was not operated on as the control. Animals were sacrificed 1, 3 days after surgery and compared to a control group. Under general intraperitoneal anaesthesia, all animals except the control group underwent pulp exposure on the occlusal face of the mandibular first molar using a 1/4-size spherical bur at high speed. The gelatin sponge with sealing medicine is placed in the perforated hole, and the cavity was sealed with glass-ionomer (Fuji, GC Corporation, Tokyo, Japan). Upon decapitation, the jaws were immersed promptly in $4 \%$ formaldehyde, embedded in paraffin, and sectioned at 5-mm thickness.

\section{Immunohistochemical Staining}

Immunostaining was performed on formalin-fixed, paraffin-embedded tissue. For immunohistochemistry, paraffin sections were dewaxed in xylene, rehydrated with distilled water, and then subjected to antigen retrieval for $30 \mathrm{~min}$ at $95^{\circ} \mathrm{C}$. The slides were subsequently incubated overnight at $4^{\circ} \mathrm{C}$ with rabbit antiCollagen I antibody (1:400,bioss,Beijing,China).Slides were then treated with an anti-rabbit secondary antibody(Zhongshanjinqiao, Beijing, China)and developed using avidin-conjugated HRP with diaminobenzidine as a substrate (Zhongshanjinqiao , Beijing, China),followed by hematoxylin counter staining.

\section{Statistical Analysis}

Results are presented as means \pm SD of at least 3 independent biological experiments. For each experiment, we have at least 3 technical repeats. Significance was determined via one-way analysis of variance test. The difference was considered statistically significant at the $\mathrm{P}<0.05$ level.

\section{Abbreviations}

Cytokeratins CK

Extracellular matrix ECM

Jumonji domain protein 3 JMJD3

Matrix metalloproteinases MMPs

Mitogen-activated Protein Kinase MAPK

Nuclear Factor-kB NF-kB

Quantitative polymerase chain reaction q-PCR 


\section{Declarations}

\section{Ethics approval and consent to participate:}

The entire study was approved by the human research committee of Peking University School of Stomatology (ethics approval number: PKUSSIRB-201732003) and performed after written informed consent from patients was obtained.

\section{Consent for publication:}

Not applicable

\section{Availability of data and materials:}

All data generated or analysed during this study are included in this published article.

\section{Competing interests:}

The authors declare that they have no competing interests

\section{Funding:}

This work was supported by the Natural Science Foundation of China (NSFC) (grant \# 81800959).

\section{Authors' contributions:}

Jie He, Tianqian Hui and Man Qin designed the study; Jie He and Yingyi Chen performed the research . Jie He ,Yingyi Chen and Ziqi Hu analyzed the data and contributied to the search and collation of literature. Jie He wrote the manuscript. Ling Ye, Man Qin and Tianqian Hui contributed to the revision of the manuscript.

\section{Acknowledgements:}

This work was supported by the Natural Science Foundation of China (NSFC) (grant \# 81800959).

\section{References}


1. van Amerongen JP, Lemmens IG, Tonino GJ. The concentration, extractability and characterization of collagen in human dental pulp. Arch Oral Biol 1983; 28:339-45.

2. Haug SR, Marthinussen MC. Acute Dental Pain and Salivary Biomarkers for Stress and Inflammation in Patients with Pulpal or Periapical Inflammation. J Oral Facial Pain Headache 2019; 33:227-233.

3. Hahn CL, Liewehr FR. Update on the adaptive immune responses of the dental pulp. J Endod 2007; 33:773-81.

4. Staquet MJ, Durand SH, Colomb E, Romeas A, Vincent C, Bleicher F, et al. Different roles of odontoblasts and fibroblasts in immunity. J Dent Res 2008; 87:256-61.

5. Frangogiannis NG. The extracellular matrix in myocardial injury, repair, and remodeling. J Clin Invest 2017; 127:1600-1612.

6. Jain A, Bahuguna R. Role of matrix metalloproteinases in dental caries, pulp and periapical inflammation: An overview. J Oral Biol Craniofac Res 2015; 5:212-8.

7. Shin SJ, Lee JI, Baek SH, Lim SS. Tissue levels of matrix metalloproteinases in pulps and periapical lesions. J Endod 2002; 28:313-5.

8. Birkedal-Hansen $\mathrm{H}$. Role of matrix metalloproteinases in human periodontal diseases. J Periodontol 1993; 64:474-84.

9. Labrie M, St-Pierre Y. Epigenetic regulation of mmp-9 gene expression. Cell Mol Life Sci 2013; 70:3109-24.

10. Moores RC, Brilha S, Schutgens F, Elkington PT, Friedland JS. Epigenetic Regulation of Matrix Metalloproteinase-1 and -3 Expression in Mycobacterium tuberculosis Infection. Front Immunol 2017; 8:602.

11. Hui T, A P, Zhao Y, Wang C, Gao B, Zhang P, et al. EZH2, a potential regulator of dental pulp inflammation and regeneration. J Endod 2014; 40:1132-8.

12. De Santa F, Totaro MG, Prosperini E, Notarbartolo S, Testa G, Natoli G. The histone H3 lysine-27 demethylase Jmjd3 links inflammation to inhibition of polycomb-mediated gene silencing. Cell 2007; 130:1083-94.

13. De Santa F, Totaro MG, Prosperini E, Notarbartolo S, Testa G, Natoli G. The histone H3 lysine-27 demethylase Jmjd3 links inflammation to inhibition of polycomb-mediated gene silencing. Cell 2007; 130:1083-94.

14. Thompson WL, Van Eldik LJ. Inflammatory cytokines stimulate the chemokines CCL2/MCP-1 and CCL7/MCP-3 through NFkB and MAPK dependent pathways in rat astrocytes [corrected]. Brain Res 2009; 1287:47-57.

15. Zhao Y, Wang CL, Li RM, Hui TQ, Su YY, Yuan Q, et al. Wnt5a promotes inflammatory responses via nuclear factor kappaB (NF-kappaB) and mitogen-activated protein kinase (MAPK) pathways in human dental pulp cells. J Biol Chem 2014; 289:21028-39.

16. Bei $Y$, Tianqian H, Fanyuan $Y$, Haiyun L, Xueyang L, Jing Y, et al. ASH1L Suppresses Matrix Metalloproteinase through Mitogen-activated Protein Kinase Signaling Pathway in Pulpitis. Journal 
of Endodontics 2017; 43:306-314.e2.

17. Kartenbeck J, Schwechheimer K, Moll R, Franke WW. Attachment of vimentin filaments to desmosomal plaques in human meningiomal cells and arachnoidal tissue. J Cell Biol 1984; 98:107281.

18. Yoshida K, Sato K, Tonogi M, Tanaka Y, Yamane GY, Katakura A. Expression of Cytokeratin 14 and 19 in Process of Oral Carcinogenesis. Bull Tokyo Dent Coll 2015; 56:105-11.

19. Krane SM. Clinical importance of metalloproteinases and their inhibitors. Ann N Y Acad Sci 1994; 732:1-10.

20. Birkedal-Hansen H, Moore WG, Bodden MK, Windsor LJ, Birkedal-Hansen B, DeCarlo A, et al. Matrix metalloproteinases: a review. Crit Rev Oral Biol Med 1993; 4:197-250.

21. Shin SJ, Lee JI, Baek SH, Lim SS. Tissue levels of matrix metalloproteinases in pulps and periapical lesions. J Endod 2002; 28:313-5.

22. Bergenholtz G. Evidence for bacterial causation of adverse pulpal responses in resin-based dental restorations. Crit Rev Oral Biol Med 2000; 11:467-80.

23. Wahlgren J, Salo T, Teronen O, Luoto H, Sorsa T, Tjaderhane L. Matrix metalloproteinase-8 (MMP-8) in pulpal and periapical inflammation and periapical root-canal exudates. Int Endod J 2002; 35:897904.

24. Evrosimovska B, Dimova C, Kovacevska I, Panov S. Concentration of collagenases (MMP-1, -8, -13) in patients with chronically inflamed dental pulp tissue. Prilozi 2012; 33:191-204.

25. Rhim EM, Ahn SJ, Kim JY, Kim KH, Lee HW, Kim EC, et al. Stimulation of matrix metalloproteinases by tumor necrosis factor-alpha in human pulp cell cultures. J Endod 2013; 39:795-800.

26. Helder MN, Bronckers AL, Woltgens JH. Dissimilar expression patterns for the extracellular matrix proteins osteopontin (OPN) and collagen type I in dental tissues and alveolar bone of the neonatal rat. Matrix 1993; 13:415-25.

27. Luo H, Wang C, Liu M, Yin B, A P, Huang D, et al. Inhibition of SOX9 Promotes Inflammatory and Immune Responses of Dental Pulp. J Endod 2018; 44:792-799.

28. Goldberg M, Njeh A, Uzunoglu E. Is Pulp Inflammation a Prerequisite for Pulp Healing and Regeneration? Mediators Inflamm 2015; 2015:347649.

29. Cooper PR, Holder MJ, Smith AJ. Inflammation and regeneration in the dentin-pulp complex: a double-edged sword. J Endod 2014; 40:S46-51.

\section{Figures}




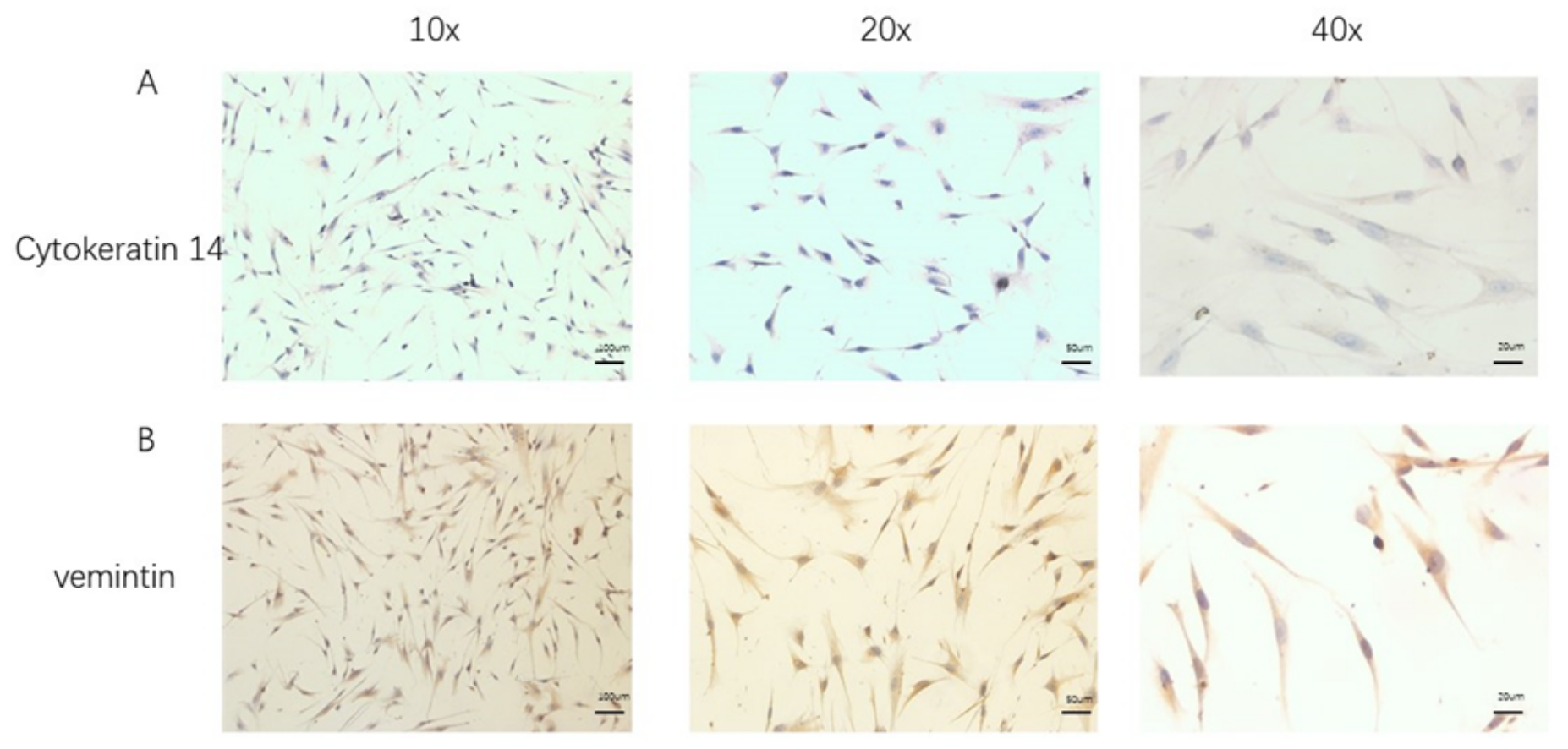

Figure 1

Expression of cytokeratin 14 and vimentin in human dental pulp cells.(A) Immunocytochemical staining using Cytokeratin14 in HDPCs showed negative staining (B) Immunocytochemical staining using vimentin in HDPCs showed positive staining. Data are representative of 3 independent experiments. scale bar:10X:100um;20X:50um;40X:20um. 
A

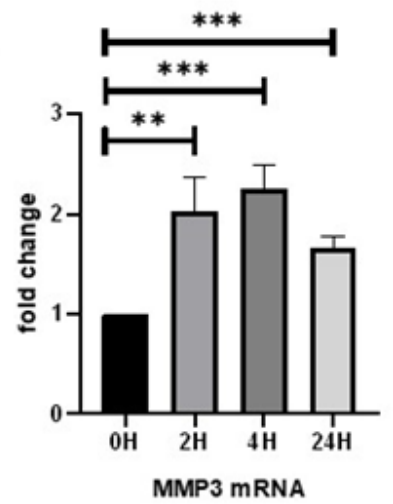

$E$

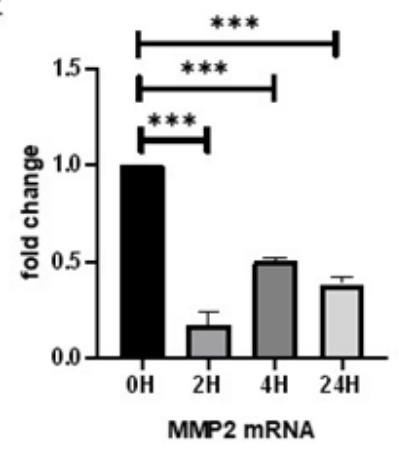

B

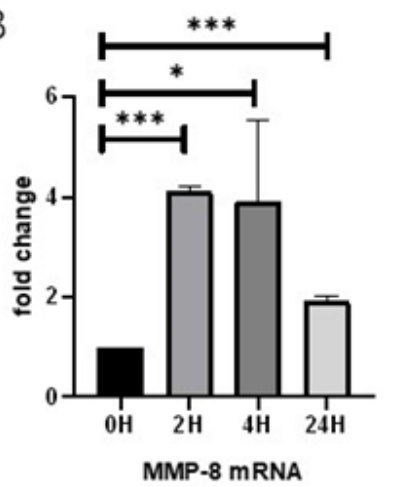

$\mathrm{F}$

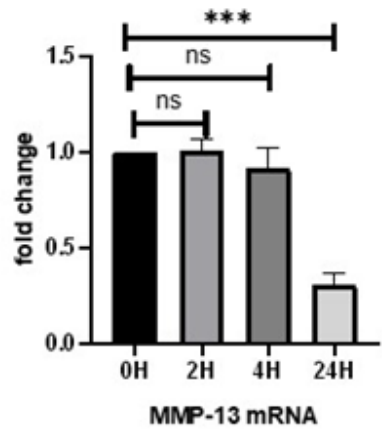

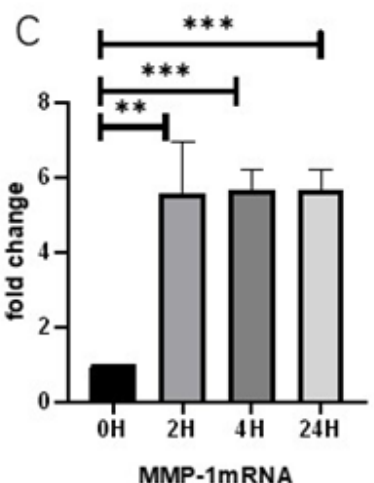

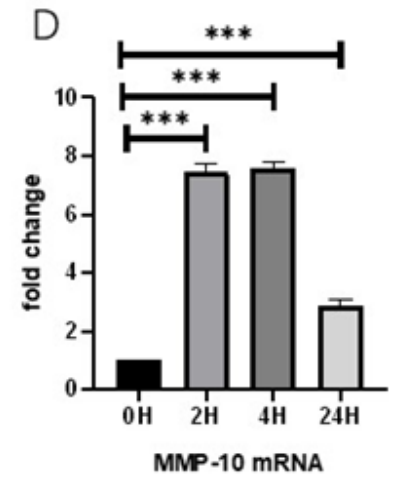

$\mathrm{H}$

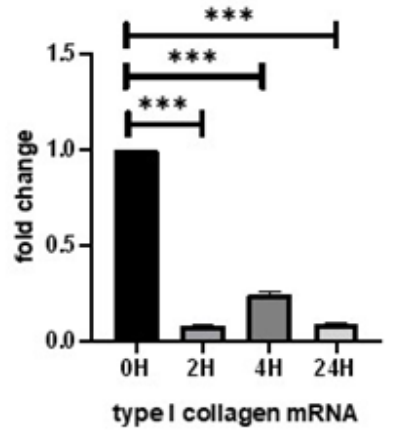

Figure 2

The effects of the EZH2 on the expression of MMPs and type I collagen in HDPCs. The effects of EZH2

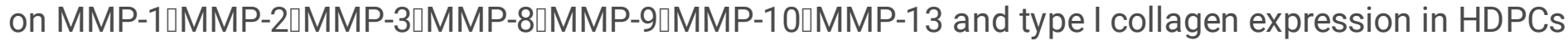
were evaluated by quantitative polymerase chain reaction. *Indicates significant difference from control. ${ }^{*} \mathrm{P}<.05,{ }^{*} \mathrm{P}<.01 .{ }^{* \star *} \mathrm{P}<.001$. 
A

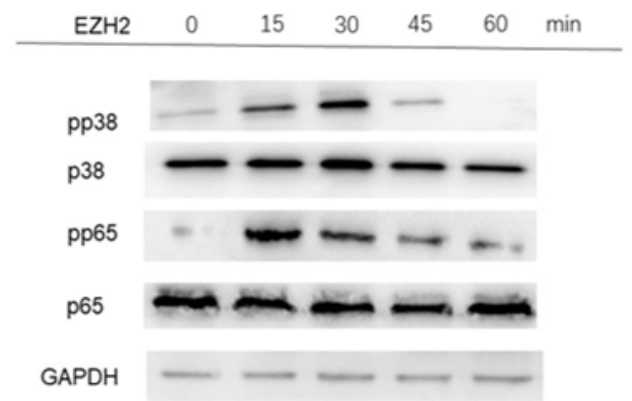

B

C
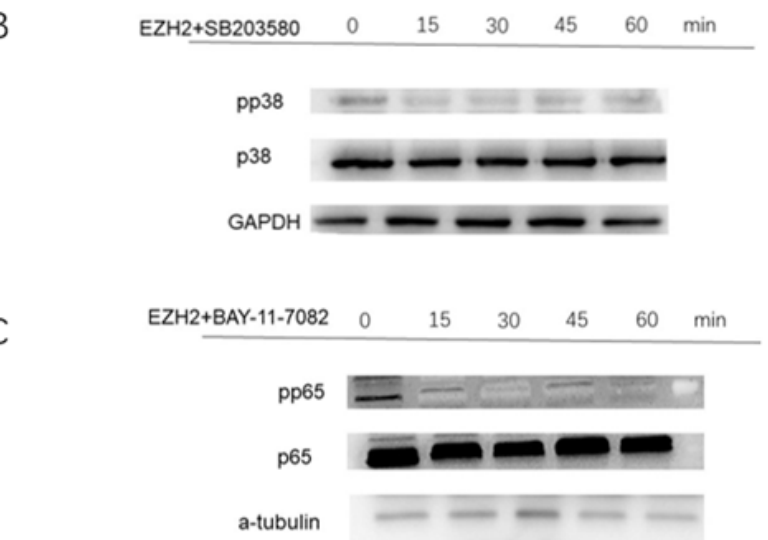
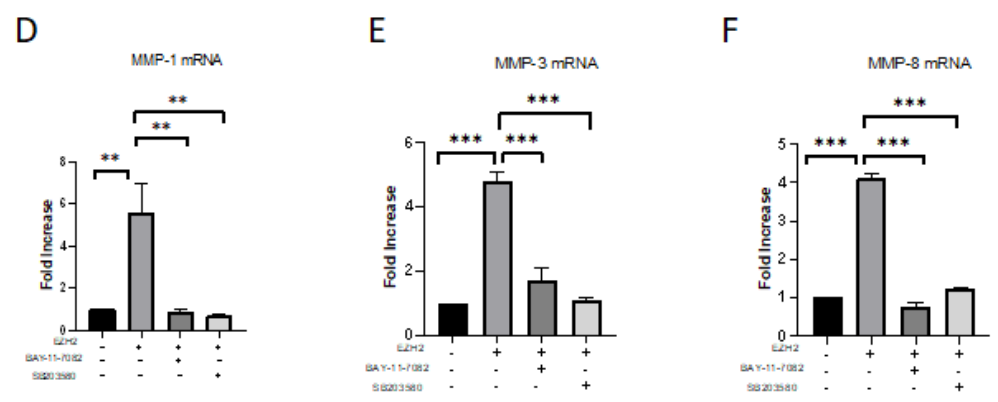

G
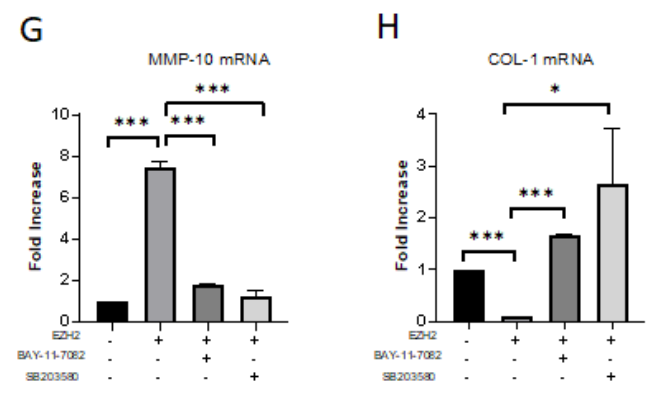

\section{Figure 3}

EZH2 activates MAPK pathway and NF-kB pathway. (A) HDPCs were incubated for the indicated time points with EZH2, p38 and NF-kB activation was assessed by Western blot analysis of total and phosphorylated signaling proteins. ( $B$ and $C$ ) HDPCs were incubated for the indicated time points with EZH2 and SB203580(a p38 inhibitor) or BAY-11-7082(an NF-kB inhibitor), p38 and NF-kB inhibition was assessed by Western blot analysis of total and phosphorylated signaling proteins. GAPDH and a-tubulin was used as a control.pp65,phosphop65; pp38,phospho-p38.(D-H)HDPCs were preincubated with BAY117082 or SB203580 before treatment with EZH2.Real-time PCR of MMPs and Col-1 expression are also shown. 

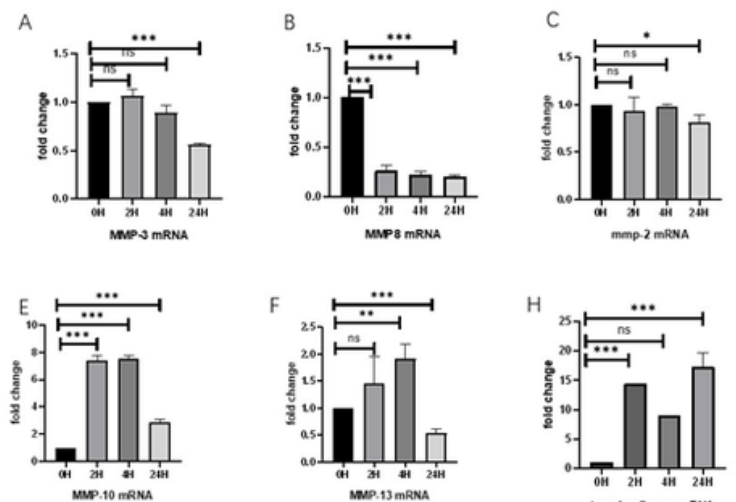

control
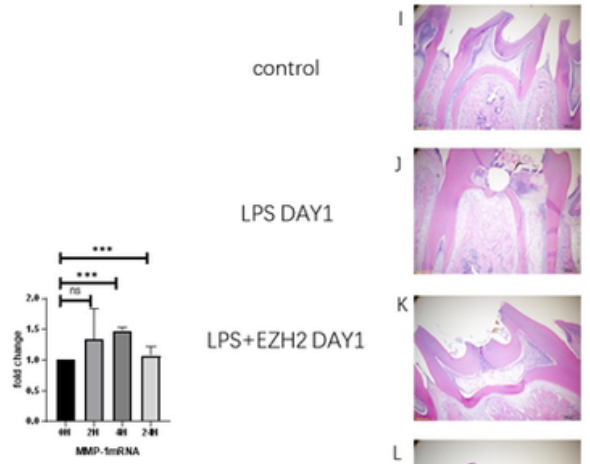

LPS DAY1

LPS+EZH2 DAY1
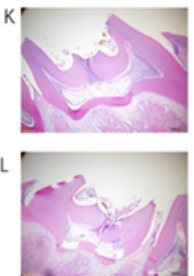

LPS+EI1 DAY1

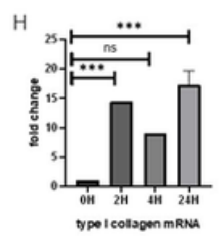

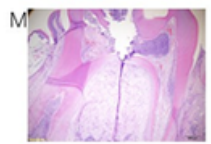

LPS+EZH2 DAY3

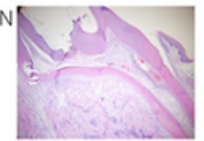

LPS+EI1 DAY3
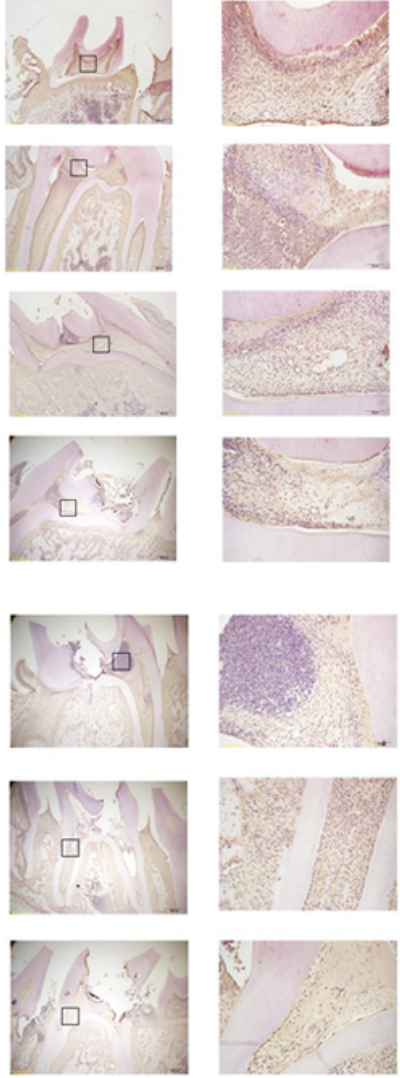

\section{Figure 4}

The effect of EZH2 and El1 on the development of pulpitis. (A-H)The effects of El1 on MMP-1DMMP-2

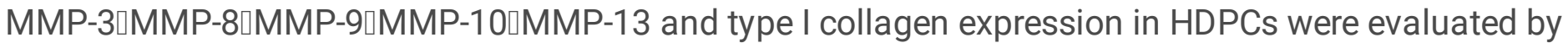
quantitative polymerase chain reaction. *Indicates significant difference from control. ${ }^{*} \mathrm{P}<.05, * * \mathrm{P}<.01$. $\star \star \star \mathrm{P}<.001$. Immunohistochemical staining showed expression of Col-1 in dental pulp in different group (I-O). scale bar:10X:100um;20X:50um;40X:20um. 